Ciência Florestal, Santa Maria, v. 3, n. 1, p. 171-184, 1993

ISSN 0103-9954

\title{
INFLUÊNCIA DA INTENSIDADE DO DESBASTE SOBRE O CRESCIMENTO EM ALTURA DE Pinus elliotti E.
}

\section{THE EFFECT OF THINNING INTENSITY ON THE HEIGHT GROWTH OF THE Pinus elliotti E.}

\author{
Paulo Renato Schneider ${ }^{1}$ César Augusto G. Finger ${ }^{1}$
}

\section{RESUMO}

O presente trabalho estudou a influência da intensidade do desbaste sobre o desenvolvimento da altura média de Pinus elliotti E., submetido a 4 (quatro) tratamentos de desbastes 25, 50 e 75\% da área basal da testemunha sem desbaste, além da testemunha sem desbaste. Os resultados obtidos permitiram concluir que a intensidade de desbaste influi no desenvolvimento da altura média e não no da altura dominante. Palavras-chave: desbaste; crescimento; altura; Pinus elliotti.

\begin{abstract}
the present research studied the effect of thinning intensity on the average and dominant height growth on a population of Pinus elliotti E., submitted to 4 thinning treatments: 25,50 e $75 \%$ of the witness basal area, and the witness with to thinning. The results obtained allow us to conclude the thinning intensity has an influence on the average height growth of Pinus elliotti E. however, it has no influence on the dominant height growth of this specie.
\end{abstract}

Keywords: thinning; growth; height; Pinus elliotti.

1 Engenheiro Florestal, Dr., Professor Titular do Departamento de Ciências Florestais, Centro de Ciências Rurais, Universidade Federal de Santa Maria, Av. Roraima, 1000, CEP 97105-900, Santa Maria (RS), Brasil. 


\section{INTRODUÇÃO}

A classificação da produção florestal pode ser efetuada com base me elementos dendrométricos como altura, área basal, volume, incremento médio em volume e altura. Destes, a altura é a variável mais utilizada devido a sua alta relação com a sua produção volumétrica e o peso de madeira.

O elemento dendrométrico altura foi proposto na Alemanha no século XVIII, como o mais apropriado para a classificação do sítio.

Esta preferência deve-se ao condicionamento da altura a fatores de natureza hereditária, e por ser menos influenciada pela densidade do povoamento do que as demais variáveis dendrométricas. A atividade fotossintética parece ter pouca influência direta no desenvolvimento da altura, pois seu crescimento processa-se sobretudo às custas das reservas de hidratos de carbono acumulados nas folhas velhas e ramos finos.

Por outro lado, um fator que deve ser considerado é que a altura dominante ou a altura média são de fácil obtenção, com baixos custos, e mostram alta correlação com a produção, o que se traduz como um fundamento importante para a classificação de sítios e construção das tabelas de produção.

Devido a isto, o presente trabalho teve por objetivo estudar a influência da intensidade do desbaste sobre o crescimento da altura média e dominante em diferentes idades de um povoamento de Pinus elliotti.

\section{REVISÃO BIBLIOGRÁFICA}

$\mathrm{Na}$ literatura florestal, a altura dominante é considerada um bom indicador da capacidade de produção do sítio. Esta variável tem sido largamente empregada para este fim, pois apresenta 
grande probabilidade de ter sempre pertencido ao estrato superior da floresta, não ser influenciada por desbastes por baixa e ser independente da densidade do povoamento. Ao contrário, a altura média, que em alguns casos é empregada para quantificar o sítio, sofre influência da densidade e é, imediatamente, alterada após o desbaste, sem que a qualidade do sítio tenha sido modificada.

Assmann (1970) relata reações diferentes sobre o crescimento em altura entre folhosas e coníferas ocasionados pela retirada de árvores em um desbaste. Segundo o autor, como regra geral, desbastes moderados proporcionam um melhor crescimento em altura e aberturas severas do dossel proporcionam um aumento do incremento em diâmetro sem aumentar o incremento em altura. Em folhosas, a abertura do dossel pode provocar o crescimento dos ramos da periferia da copa em detrimento do incremento em altura como observado em Fagus silvatica.

Segundo Smith \& Strub (1991), povoamentos muito abertos ou muito densos afetam o desenvolvimento da altura dominante. O autor constatou em experimento com Pinus taeda e Pinus elliotti, em espaçamentos variando entre $1,8 \times 1,8$ a $3,7 \times 3,7$ e $1,8 \times 1,8$ a 4,6 x 4,6 metros, respectivamente, que o crescimento em altura dominante decresceu nos espaçamentos fechados e a diferença na altura aumentou quando as árvores ficaram mais velhas. Para $P$. taeda ocorreu diferença de $1,1 \mathrm{~m}$ de altura entre os espaçamentos de $1,8 \times 1,8$ e $3,7 \times 3,7 \mathrm{~m}$, aos 15 anos e $1,5 \mathrm{~m}$ aos 30 anos. Para P. elliotti, a diferença entre $1,8 \times 1,8$ e $4,6 \times 4,6 \mathrm{~m}$ foi de $1,5 \mathrm{~m}$ aos 15 anos, e 3,4 $\mathrm{m}$ aos 30 anos. Os resultados sugerem que, em espaçamentos mais apertados que 2,4 x 2,4 m, o P. taeda começa a sofrer forte redução do desenvolvimento da altura dominante, o mesmo acontece para $P$. elliotti a partir de $3,0 \times 3,0 \mathrm{~m}$ de espaçamento.

Schoenau (1969) estudou a influência da densidade em povoamento de Acacia mearnsii sobre o crescimento em altura, 
volume e produção de casca em um experimento com nove intensidades de desbastes não observando diferença estatística da densidade do povoamento sobre a altura, aos 9 anos.

Com o mesmo objetivo, Schoenau (1973) efetuou a análise de uma série de experimentos como instalado no Kenya em nove sítios, sendo os tratamentos definidos pela densidade populacional do povoamento. No final de 10 anos, os resultados das regressões da altura média com a densidade do povoamento foram significativos em dois sítios, constatando a influência da densidade do povoamento sobra a altura média. Entretanto, o autor diz que esta influência pode ser negligenciada por ser mínima.

Van Laar (1973) analisou o crescimento em altura de Pinus radiata, em um experimento com três graus de desbaste e três intensidades de poda. Após um período de 8 anos de observação, constatou que a intensidade de poda afeta a taxa de crescimento em altura, concluindo que quanto mais alta for a poda, menor é o crescimento em altura e a taxa de crescimento que, nestas condições, decresce de forma curvilinar.

Hummel (1947), apud Reinstorf (1970), comparou o crescimento em altura de Norway spruce submetido a quatro graus de desbaste, como desbaste fraco seletivo por baixo; desbaste moderado/forte por baixo; desbaste forte por baixo; desbaste fraco clímax. Os resultados demostraram que o incremento em altura dominante não foi afetado nos quatro regimes de desbaste e a taxa de crescimento em altura foi uniforme no período observado.

Reinstorf (1970) estudou a relação da densidade de plantas sobre o desenvolvimento da altura de Pinus elliotti em um experimento CCT (Correlated Curve Trend) de desbaste, repetido em 3 sítios, não encontrando evidências estatísticas de influência da densidade do povoamento sobre a taxa de crescimento em altura. Em um dos locais estudados detectou uma pequena influência da densidade do povoamento sobre o crescimento em altura, 
atribuindo-a, entretanto, à heterogeneidade do solo.

\section{MATERIAL E MÉTODOS}

Os dados utilizados no presente trabalho têm origem em um experimento de desbaste de área basal, instalado na Empresa Seiva S.A - Florestas e Indústrias, localizada em Curitibanos, no Estado de Santa Catarina. A área experimental fica situada entre as coordenadas geográficas de $27^{\circ} 07^{\prime}$ de latitude sul e $50^{\circ} 26^{\prime}$ de longitude oeste do meridiano de Greenwich.

Esta região localiza-se no Planalto Central Catarinense, em uma formação geográfica basáltica, com relevo ondulado. A altitude é variável, situando-se em torno de 1.000 metros do nível do mar.

Segundo a classificação de Koeppen, a região é caracterizada por um clima $\mathrm{Cfb}$, mesotérmico, subtropical úmido, com verões frescos, se estação seca definida e geadas frequentes. A temperatura média das máximas de $21,3^{\circ} \mathrm{C}$ e das mínimas de $12,3^{\circ} \mathrm{C}$. A temperatura média anual está em torno de $15,4^{\circ} \mathrm{C}$, umidade relativa do ar de $66,1 \%$, precipitação média anual de aproximadamente $1.745 \mathrm{~mm}$, regularmente distribuídas durante o ano (MOTA et al., 1971).

A região apresenta uma grande variedade dos solos, predominando os solos do tipo bruno, ácidos, presumivelmente uma modalidade altimontana de solos do tipo latossolo e litólicos e solos humíferos de vales altimontanos (CAMARGO \& BEREMA, 1966).

O experimento foi instalado em março de 1976, em um povoamento de Pinus elliotti, com idade de 7,5 anos, implantado em espaçamentos de $2,0 \times 2,0$ metros. 
O delineamento experimental utilizado foi o de blocos casualizados em parcelas subdivididas, com quatro tratamentos e duas repetições. Cada parcela ocupava uma superfície de 1.000 $\mathrm{m}^{2}$ e os tratamento definidos em relação à área basal máxima da testemunha, sem desbaste. Foram tomados os tratamentos com desbaste até 25,50 e $75 \%$ da área basal da testemunha, além da própria testemunha sem desbaste. Os dados utilizados foram a altura média e a dominante por idade. A idade de medição foi utilizada para definir as subparcelas, representadas pelas observações nas idades de 7,5 a 21,5 anos, ou seja, totalizando 15 subparcelas.

O modelo matemático para explicar o crescimento em altura foi exposto por:

$$
Y i j k=U+B i+T j+S i j+N k+(T N) j k+E i j k
$$

Em que: Yijk = variável resposta (altura); $\mathrm{U}=$ média geral; $\mathrm{Bi}=$ efeito das repetições; $\mathrm{Tj}=$ efeito principal das parcelas nos tratamentos j (densidade); $\mathrm{Sij}=$ erro do experimento para o local de tratamentos; $\mathrm{Nk}=$ efeito das subparcelas nos tratamentos $\mathrm{k}$ (idade); (TN) jk = interação entre os fatores T e N; Eijk = erro experimental associado com as subparcelas.

O crescimento em altura média e dominantes foram ajustados como função da idade através do modelo de Backmann, expresso por:

$$
\text { In } h=\text { bo }+ \text { bi. In } t+b 2 . \operatorname{In}^{2} t
$$

Em que: $\mathrm{h}=$ altura média ou dominante em metros; $\mathrm{t}=$ idade em anos; 
Os cálculos da análise da variância do delineamento utilizado foram realizados através da PROC ANOVA do Pacote SAS, em computador IBM 3090 da Universidade Federal de Santa Maria.

\section{RESULTADO E DISCUSSÃO}

Os resultados da análise estatística da influência de intensidade do desbaste definida pelos pesos de $0,25,50$ e $75 \%$ de área basal da testemunha, tratamentos de $0,1,2,3$, respectivamente, sobre o crescimento da altura média do povoamento encontram-se na Tabela 1.

TABELA 1: Análise de variância da altura média em função da intensidade do desbaste.

TABLE 1: Analysis of variance in the mean height because of the thinnning intensity.

\begin{tabular}{l|c|r|c|c|c}
\hline Fonte de varição & GL & \multicolumn{1}{c|}{ SQ } & QM & F & Prob>F \\
\hline Bloco & 1 & 3,2308 & 3,2308 & & \\
Tratamentos (A) & 3 & 19,6162 & 6,5387 & 5,81 & 0,0912 \\
Resíduo (a) & 3 & 3,3769 & 1,1256 & & \\
Idade (B) & 14 & $2.360,90$ & 168,63 & $2.913,7$ & 0,0001 \\
Interação (A*B) & 42 & 9,2086 & 0,2192 & 3,79 & 0,0001 \\
Resíduo (b) & 56 & 3,2409 & 0,0578 & & \\
TOTAL & 119 & $2.399,60$ & & & \\
\hline
\end{tabular}

Os resultados obtidos mostram a existência de diferença significativa entre tratamentos em nível de probabilidade $>\mathrm{F}=0,0912$, demostrando que a intensidade de desbaste influencia no desenvolvimento da altura média. 
Este efeito é explicado pelo aumento da altura média imediatamente após o desbaste por baixo, sem que existam mudanças na qualidade do sítio.

O efeito dos tratamentos aplicados em diferentes ocasiões (idade do povoamento) sobre o desenvolvimento da altura média foi significativo em nível de probabilidade $>F=0,0001$, evidenciando que $\mathrm{o}$ crescimento em altura responde de maneira diferente à intensidade de desbaste aplicada ao longo do tempo.

A Figura 1 mostra as tendências de desenvolvimento da altura média para os tratamentos de desbaste entre as idades de 7 a 22 anos. Observa-se que no período de 7 a 11 anos existe uma sobreposição das curvas de altura média e, que a partir desta idade iniciou-se uma diferenciação destas tendências, nas quais o tratamento 2 mostra as maiores alturas médias.

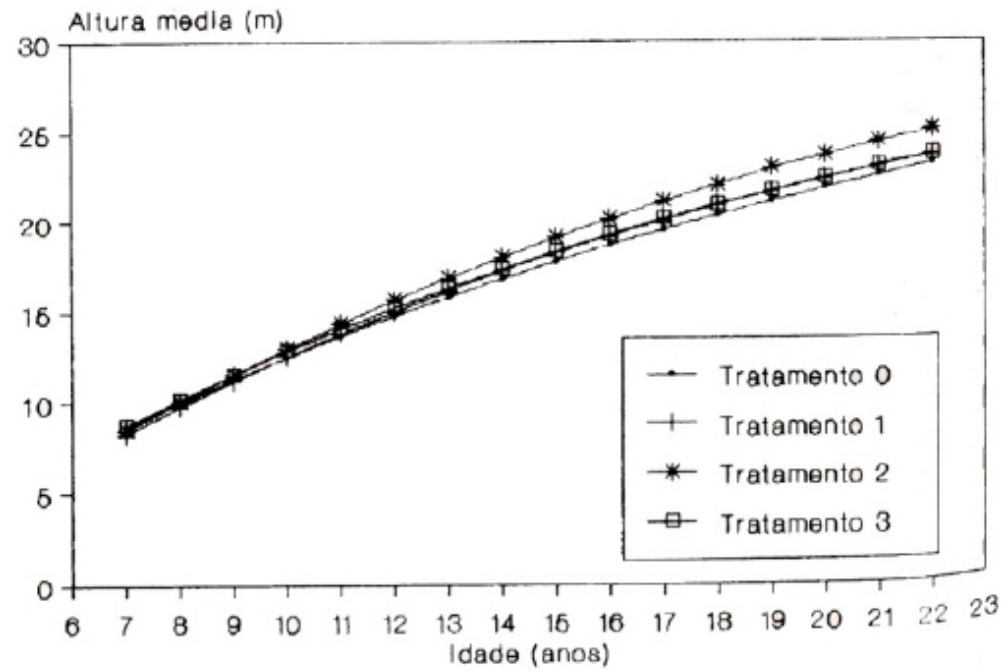

FIGURA 1: Crescimento em altura média em função da idade por intensidade de desbaste.

FIGURE 1: Growth in the mean height because of age by thinning intensity. 
Influência da intensidade do desbaste sobre o crescimento em altura...179

As curvas apresentadas na Figura 1 foram ajustadas pela equação de Backmann cujos coeficientes e precisão estatística encontram-se na Tabela 2.

TABELA 2: Coeficientes e precisão das equações para a variável dependente altura média e dominante, por tratamento.

TABLE 2: Coefficients and precision of equations for the variable depending on the mean and dominant height, per treatment.

\begin{tabular}{c|c|c|c|c|c|c|c}
\hline \multirow{2}{*}{$\begin{array}{c}\text { Variável } \\
\text { Dependente }\end{array}$} & \multirow{2}{*}{ Tratamento } & \multicolumn{7}{c}{ Coeficientes } \\
\cline { 3 - 8 } & & $\mathrm{b} 0$ & $\mathrm{~b} 1$ & $\mathrm{~b} 2$ & $\mathrm{R}^{2}$ & $\mathrm{~S} y x$ & $\mathrm{CV} \%$ \\
\hline \multirow{3}{*}{ Altura } & 0 & $-0,9356$ & 2,0450 & $-0,2345$ & 0,99 & 0,0287 & 1,03 \\
Média & 1 & $-1,6010$ & 2,5331 & $-0,3207$ & 0,99 & 0,0272 & 0,97 \\
& 2 & $-1,6329$ & 2,5625 & $-0,3208$ & 0,99 & 0,0214 & 0,75 \\
& 3 & $-1,2096$ & 2,2771 & 0,2786 & 0,99 & 0,0176 & 0,63 \\
\hline \multirow{2}{*}{$\begin{array}{c}\text { Altura Do- } \\
\text { minante }\end{array}$} & 1 & $-1,2352$ & 2,4205 & $-0,3194$ & 0,99 & 0,0273 & 0,94 \\
& 2 & $-0,6005$ & 1,8963 & $-0,2119$ & 0,99 & 0,0231 & 0,79 \\
& 3 & $-1,1435$ & 2,2806 & $-0,2803$ & 0,99 & 0,0133 & 0,46 \\
\hline
\end{tabular}

\section{Efeito do desbaste sobre a altura dominante}

Os efeitos da intensidade do desbaste dos tratamentos de 0 , 1,2 e 3 sobre a variável altura dominante não foram significantes estatisticamente em nível de probabilidade $>F=0,2681$, indicando que a altura dominante não foi influenciada pelos tratamentos, conforme a Tabela 3.

Os efeitos dos tratamentos nas diferentes idades, representado pela interação, foi significante em nível da probabilidade $>\mathrm{F}=0.0040$, indicando que no período estudado, houve respostas diferentes da altura dominantes aos tratamentos. 
TABELA 3: Análise de variância da altura dominante em função da intensidade de desbaste.

TABLE 3: Analysis of the variance of dominant height because of the thinning intensity.

\begin{tabular}{lccc|c|c}
\hline Fonte de variação & GL & SQ & QM & F & Prob.>F \\
\hline Bloco & 1 & 0,0182 & 0,0182 & & \\
Tratamentos (A) & 3 & 6.4785 & 2,1595 & 2,19 & 0,2681 \\
Resíduo (a) & 3 & 2.9575 & 0,9858 & & \\
Idade (B) & 14 & $2.360,90$ & 168,63 & $1.450,8$ & 0,0001 \\
Interação (A*B) & 42 & 10,4473 & 0,2487 & 2,14 & 0,0040 \\
Resíduo (b) & 56 & 6,5072 & 0,1162 & & \\
Total & 119 & $2.3870,20$ & & & \\
\hline
\end{tabular}

Observa-se na Figura 2 que as curvas de altura dominante sobre a idade para os 4 tratamentos apresentaram valores semelhantes. No entanto, as alturas observadas no tratamento 3 foram ligeiramente inferiores às das demais no período de 11 a 20 anos, igualando-se às alturas dominantes nos tratamentos 1 e 2 a partir desta idade.

Observa-se também que a altura dominante no tratamento sem desbastes (tratamento 1) foi coincidente, até os 17 anos, com as alturas dos tratamentos 1 e 2 e que a partir desta idade apresentou maior desenvolvimento. Esse efeito pode estar indicando alta concorrência entre as árvores e o agravamento das condições do sítio.

As curvas representadas na Figura 2 foram ajustadas pela função de Backmann, apresentada na metodologia, cujos coeficientes e estatísticas de precisão podem ser vistos na Tabela 2. 


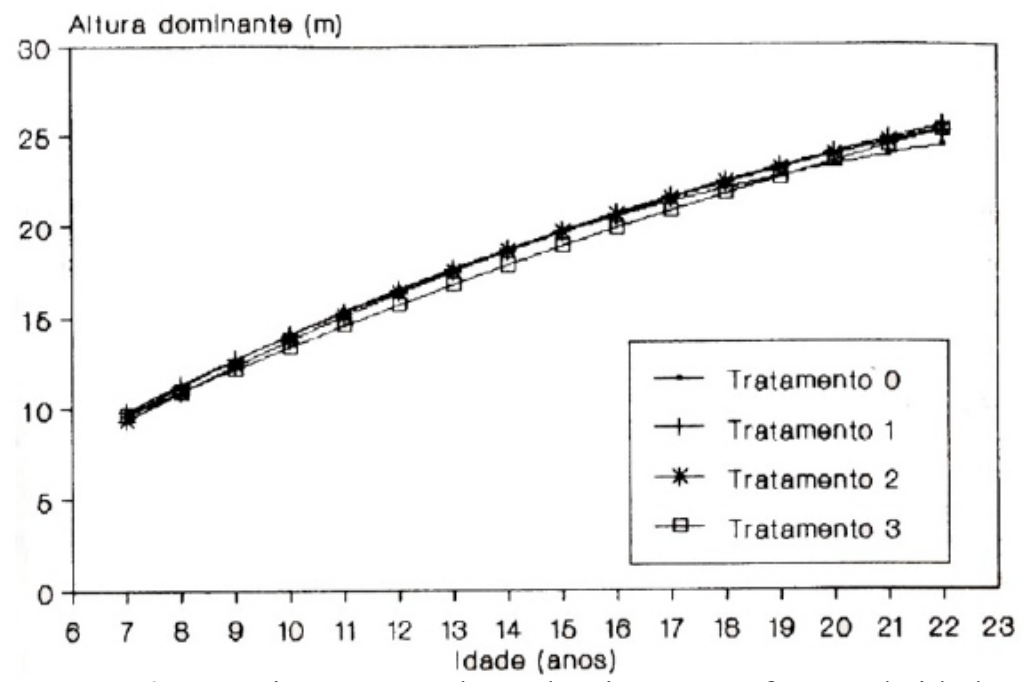

FIGURA 2: Crescimento em altura dominante me função da idade por intensidade de desbaste.

FIGURE 2: Growth in the dominant height because of the age by thinning intensity.

Embora fosse esperado que o tratamento de maior intensidade de desbaste (tratamento com $75 \%$ da área basal da testemunha) apresentasse as maiores alturas médias e a menor diferença com a altura dominante, pois é o tratamento com menor número de árvores remanescentes, isto não ocorreu.

Este efeito, no entanto, foi evidente no tratamento com peso de desbaste de $50 \%$ da área basal da testemunha; o qual também mostrou as maiores alturas médias entre os tratamentos testados.

A análise da diferença entre as alturas dominantes para cada um dos tratamentos permitiu concluir a Figura 3, a qual mostra o tratamento 3 como o de menor diferença entre a altura dominante e a média até a idade de 15 anos, sendo a partir desta idade superado pelo tratamento 2 . 
Essas tendências parecem resultar de um efeito semelhante ao descrito por Assmann (1970) para o crescimento da área basal. Por este efeito, povoamentos jovens, submetidos a desbastes fortes, sofrem uma aceleração do crescimento gerando incrementos volumétricos maiores que no povoamento não desbastado.

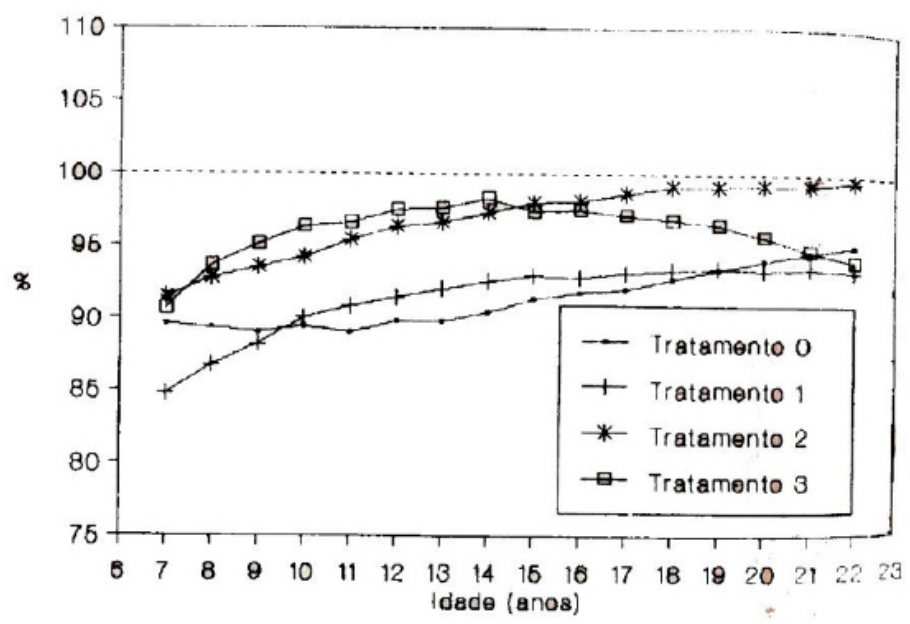

FIGURA 3: Percentagem da altura média em relação à altura dominante por idade e intensidade de desbaste.

FIGURE 3: Percentage of the mean height in relation to the dominant height per age and thinning intensity.

O mesmo grau de desbaste aplicado em povoamentos adultos e velhos leva à redução do incremento volumétrico. Este efeito de redução do incremento em altura foi também relatado por Assmann (1970) e Smith Strub (1991). 
Influência da intensidade do desbaste sobre o crescimento em altura...183

\section{CONCLUSÕES}

Dos resultados obtidos no presente trabalho é possível afirmar que:

a) $\mathrm{O}$ desenvolvimento da altura média é influenciado pela intensidade de desbaste;

b) A média das alturas no tempo foram estatisticamente iguais entre os tratamentos 2, 3, 1 e entre os tratamentos 3,1 e 0, apresentando, entretanto, uma diferença entre a maior e a menor altura de 1,0 metro;

c) O desenvolvimento da altura dominante não foi influenciado pela intensidade de desbaste, apesar de que a partir da idade de 17 anos, houvesse a mudança na tendência das curvas de altura dos tratamentos;

d) Ainda que a análise estatística tenha mostrado diferença significante no efeito dos desbastes e idade, sobre o desenvolvimento das alturas médias e dominantes, a variação das alturas dominantes foi menor do que a observada na altura média, indicando que a altura dominante deve ser preferida à altura média para estudos de sítio e produção.

\section{REFERÊNCIAS BIBLIOGRÁFICAS}

ASSMANN, E. The principles of forest yield study. Oxford: Pergamon Press, 1970. 506p.

CAMARGO, M.N.; BEREMA, J. Delineamento esquemático dos solos do Brasil. Rio de Janeiro: DPFS - Ministério da Agricultura, 1966. 200p. (Boletim Técnico, 1).

HUMMEL, F.C. Bowmont Norway spruce sample plots. Forestry, v.21, 1947.

MOTA, F.S.; BEINSDORF, M.I.C.; GAECEZ, J.R.B. Zoneamento Agro-climatico do Rio Grande do Sul e Santa Catarina. Pelotas: Ministério da Agricultura, 1971.90p.

REINSTORF, L.O. The Influence of stand density on growth of Pinus elliotti. Stellenbosch : University of Stellenbosch, 1970. 62p. SCHOENAU, A.P.G. A site evaluation study in Black wattle (Acacia mearnsii De Wild.). Stellenbosch: Univ. von Stellenbosch, v. 44, Serie A, n.2, 1969. 214p. 
SCHOENAU, A.P.G. Height growth and site index curves for Acacia mearsii on the Uasin Gishu Olanteau of Kenya. Commonwealth Forest Review, v.52, n.53, p.245-253, 1973.

SMITH, W.D.; STRUB, M.R. Initial Spacing: How Many Trees to Plant. In: FOREST REGENERATION MANUAL. London: Kluwer Acad. Pub, 1991. p. 281-320.

VAN LAAR, A. Needle-Biomass, Growth and Growth Distribuition of Pinus radiate in South Africa in Relation to Pruning and Thinning. Muenchen: Forstliche Forschungsanstalt, 1973. 282p. 